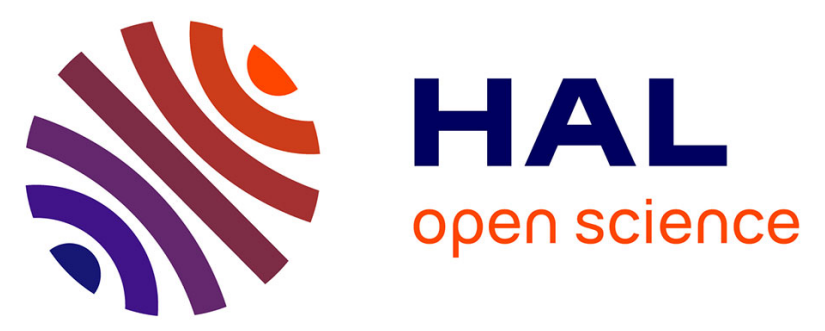

\title{
Climbing Jacob's Ladder of Structural Refinement: Introduction of a Localized Molecular Orbital-Based Embedding for Accurate X-ray Determinations of Hydrogen Atom Positions
}

Erna K Wieduwilt, Giovanni Macetti, Alessandro Genoni

\section{To cite this version:}

Erna K Wieduwilt, Giovanni Macetti, Alessandro Genoni. Climbing Jacob's Ladder of Structural Refinement: Introduction of a Localized Molecular Orbital-Based Embedding for Accurate X-ray Determinations of Hydrogen Atom Positions. Journal of Physical Chemistry Letters, 2020, 12, pp.463471. 10.1021/acs.jpclett.0c03421 . hal-03089861

\section{HAL Id: hal-03089861 \\ https://hal.univ-lorraine.fr/hal-03089861}

Submitted on 28 Dec 2020

HAL is a multi-disciplinary open access archive for the deposit and dissemination of scientific research documents, whether they are published or not. The documents may come from teaching and research institutions in France or abroad, or from public or private research centers.
L'archive ouverte pluridisciplinaire HAL, est destinée au dépôt et à la diffusion de documents scientifiques de niveau recherche, publiés ou non, émanant des établissements d'enseignement et de recherche français ou étrangers, des laboratoires publics ou privés. 
This document is the Accepted Manuscript version of a Published Work that appeared in final form in The Journal of Physical Chemistry Letters, copyright (C) American Chemical Society after peer review and technical editing by the publisher. To access the final edited and published work see https://doi.org/10.1021/acs.jpclett.0c03421 


\title{
Climbing Jacob's Ladder of Structural Refinement: \\ Introduction of a Localized Molecular Orbital-Based
}

\section{Embedding for Accurate X-ray Determinations of}

\section{Hydrogen Atoms Positions}

\author{
Erna K. Wieduwilt, ${ }^{\text {a }}$ Giovanni Macetti, ${ }^{\text {a }}$ Alessandro Genoni ${ }^{\text {a, }}{ }^{*}$ \\ a Université de Lorraine \& CNRS, Laboratoire de Physique et Chimie Théoriques (LPCT), \\ UMR CNRS 7019, 1 Boulevard Arago, F-57078, Metz, France
}

\begin{abstract}
Positions of hydrogen atoms in molecules are fundamental in many aspects of chemistry. Nevertheless, most of molecular structures are obtained from refinements of X-ray data exploiting the independent atom model (IAM), which uses spherical atomic densities and provides bond lengths involving hydrogen atoms that are too short compared to the neutron reference values. To overcome the IAM shortcomings, the wave function-based Hirshfeld atom refinement (HAR) method has been recently proposed, emerging as a promising strategy able to give element-hydrogen bond distances in excellent agreement with the neutron ones in terms of accuracy and precision. In this Letter, we propose a significant improvement of HAR based on the idea of describing the crystal environment explicitly in the underlying wave function calculation through a quantum mechanical embedding strategy that exploits extremely localized molecular orbitals. Test-bed refinements on a crystal structure characterized by strong intermolecular interactions are also discussed.
\end{abstract}




\section{TOC GRAPHICS}

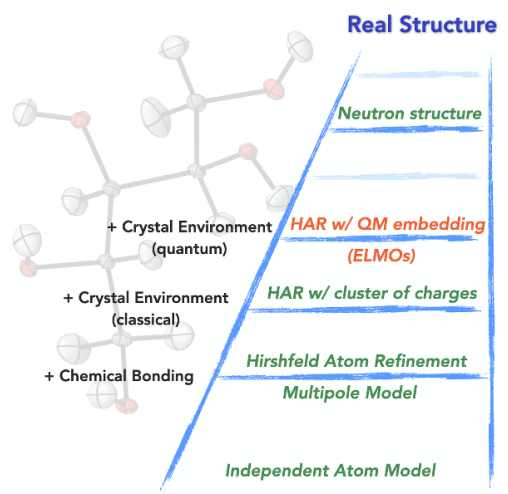

KEYWORDS: X-ray structural refinement, Quantum Crystallography, Hirshfeld Atom Refinement, Extremely Localized Molecular Orbitals, QM/ELMO embedding technique, hydrogen atoms positions 
Nowadays experimental crystal structures are undoubtedly important points of departure to start evaluating molecular properties and to perform molecular computations or simulations. Of course, the higher the accuracy of the crystal structure, the better the starting point. In this framework, a crucial role is actually played by positions and thermal parameters of hydrogen atoms, which are fundamental in many aspects and fields of chemistry. ${ }^{1-6}$

To obtain reliable hydrogen structural parameters, one should resort to neutron diffraction, which really enables to determine hydrogen atoms positions accurately and precisely because neutrons are strongly diffracted by atomic nuclei. Nevertheless, the use of this experimental technique is intrinsically and strongly limited by the fact that it requires nuclear reactors or spallation sources. For this reason, X-ray diffraction remains the method of choice, although it is often neglected that practically almost all the X-ray-based structural determinations rely on the very basic independent atom model (IAM), ${ }^{7}$ an approximation that considers the electron density of the molecule under exam as sum of tabulated, spherical atomic electron distributions previously obtained through quantum mechanical calculations on isolated atoms. Therefore, IAM completely neglects the aspherical deformation of the atomic electron densities due to chemical bonds. Consequently, since X-rays are diffracted by electrons and hydrogen atoms have only one electron, IAM unavoidably leads to element-hydrogen (E-H) bond distances that are significantly shorter than those observed from neutron diffraction measurements.

To partially overcome this drawback the E-H bond lengths can be adjusted to tabulated and averaged neutron diffraction-based values. ${ }^{8,9}$ However, this approach lacks flexibility and cannot be applied to hydrogen atoms in particular bonding situations. Therefore, refinement strategies that enable to obtain accurate and precise E-H bond lengths specifically for systems/crystals under examination were proposed in the framework of quantum crystallography ${ }^{10-14}$ over the years. Most of them tried to account for the asphericity of the 
atomic electron distributions in chemical bonding ${ }^{15}$ through the multipole models of the electron density, ${ }^{16,17}$ exploiting pseudoatoms databanks ${ }^{18-23}$ or ad hoc multipole model parameters for the hydrogen atom corresponding to its electron density in the $\mathrm{H}_{2}$ molecule ${ }^{24}$. However, in this context, the most important step forward is represented by the more recent Hirshfeld atom refinement (HAR) method..$^{25-30}$ At each iteration of the refinement, this technique requires a quantum chemical calculation that provides a molecular electron density that is afterwards subdivided into aspherical atomic contributions by exploiting the so-called Hirshfeld stockholder partitioning approach ${ }^{31,32}$. Extensive test-bed refinements have clearly shown that HAR is generally able to provide E-H bond distances that are as accurate and precise as those obtained from neutron diffraction experiments, also with X-ray diffraction data at resolutions as low as $0.8 \AA .{ }^{28}$ However, the real quality of ADPs resulting from HARs is still unclear and, in general, they have to be taken with caution. ${ }^{33,34}$ In fact, although physically reasonable, they do not seem as accurate as those resulting from the refinements of neutron data or as those estimated through $\mathrm{SHADE}^{35,36}$ (simple hydrogen anisotropic displacement estimator).

Initially based on Hartree-Fock (HF) and density functional theory (DFT) calculations, the HAR technique has been also recently extended to post-HF multi-determinant strategies. ${ }^{37}$ Moreover, to limit its intrinsically higher computational cost and to extend it to the refinement of macromolecular crystal structures, HAR has been coupled with libraries of extremely localized molecular orbitals ${ }^{38-40}$ (ELMOs; ${ }^{41-47}$ see Supporting Information for more details about ELMOs and ELMO databanks). This gave rise to the HAR-ELMO approach ${ }^{48}$ that enabled to successfully refine crystal structures of polypeptides, proteins and also organometallic compounds at a significantly reduced computational cost compared to traditional Hirshfeld atom refinements. In the same context, it is also worth mentioning the fragHAR method, ${ }^{49}$ which combines the traditional HAR technique with the molecular 
fractionation with conjugate caps (MFCC) strategy $^{50}$ to speed up the calculations of QMbased structure factors for polypeptides and proteins.

Test calculations on HAR have also shown that taking into account the crystal environment is crucial to carry out better refinements, especially when strong intermolecular interactions (e.g., hydrogen bonds) are present. ${ }^{30}$ To accomplish this task, instead of performing expensive computations on large supermolecules that account for the existing interactions in the crystal, point charges obtained from the quantum mechanical calculation at the previous refinementstep are generally placed at symmetry-generated positions around the reference crystal unit within a given radius from any atom (default value set equal to $8 \AA$ ) ${ }^{25-27}$ This simple strategy generally speeds up the convergence of the HAR procedure and, above all, it improves the agreement with neutron results.

In this Letter, we introduce a new and significant step forward for the embedding technique in HAR. Instead of simply using a cluster of point charges, in the refinement process we propose to mimic the crystal environment of the reference unit at a fully quantum mechanical level by exploiting a novel embedding approach: the quantum mechanics / extremely localized molecular orbital (QM/ELMO) method, ${ }^{51-53}$ namely a technique that successfully enables to treat the most important (or chemically relevant) region of the system under examination at a higher level of theory (HF, DFT or post-HF levels), while the remaining part is described through frozen extremely localized molecular orbitals previously transferred from the recently constructed ELMO libraries ${ }^{40}$ or from proper tailor-made model molecules. Along this line, another possibility considered in this work is the coupling of HAR with the more recent QM/ELMO/MM technique, a three-layer approach where the outermost region is treated by means of classical Molecular Mechanics (MM). ${ }^{54}$

As in a traditional Hirshfeld atom refinement, also for the new version of the method proposed in this work, it is necessary to define a reference crystal-unit, which must 
correspond at least to the asymmetric unit of the crystal structure that one wants to refine. After this preliminary definition, the new embedded-HAR technique consists in the following self-consistent cycle:

1. Quantum chemical computation on the reference unit. In our case, instead of a simple QM calculation or a QM computation with an embedding cluster of point charges, we perform a QM/ELMO or QM/ELMO/MM calculation in which the chosen reference crystal unit is fully treated at QM level (HF, DFT or post-HF), while the crystal environment (i.e., the other symmetry-generated units within a given radius from any atom of the reference unit) is described through transferred extremely localized molecular orbitals and, in the QM/ELMO/MM case, also through a classical MM force field (see Figure 1). This allows a complete or partial quantum mechanical treatment of the embedding due to the crystal environment (see Supporting Information for more details about the QM/ELMO ${ }^{51-53}$ and QM/ELMO/MM ${ }^{54}$ methods).

2. Partitioning of the electron density into Hirshfeld atoms. The stockholder Hirshfeld partitioning technique ${ }^{31,32}$ is used to subdivide the reference-unit electron density $\rho(\boldsymbol{r})$ (i.e., the electron density of the QM region) obtained at the previous step into aspherical atomic contributions $\left\{\rho_{A}(\boldsymbol{r})\right\}$ according to the following equation:

$$
\rho_{A}(\boldsymbol{r})=w_{A}(\boldsymbol{r}) \rho(\boldsymbol{r})
$$

where $w_{A}(\boldsymbol{r})$ is the Hirshfeld partitioning function for the generic atom $A$. This is given by the spherically averaged atomic density $\rho_{A}^{o}(\boldsymbol{r})$ of atom $A$ divided by the sum of the spherically averaged densities associated with the atoms in the reference crystal-unit (namely, divided by the "promolecular" density of the reference crystal-unit):

$$
w_{A}(\boldsymbol{r})=\frac{\rho_{A}^{o}(\boldsymbol{r})}{\sum_{B=1}^{\text {Natoms }} \rho_{B}^{o}(\boldsymbol{r})}
$$




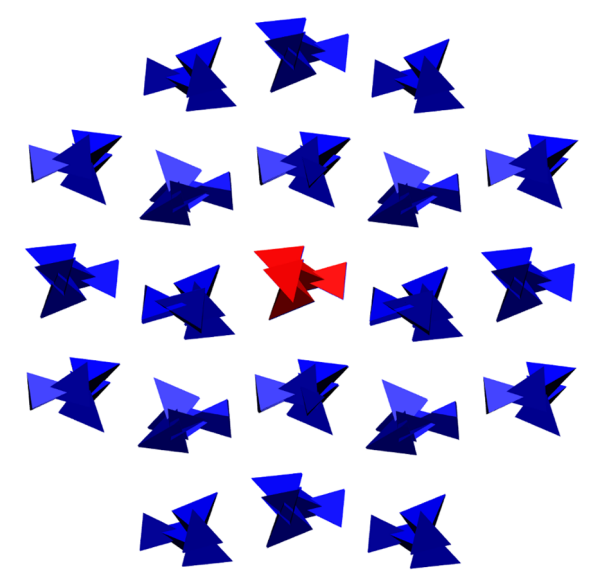

A

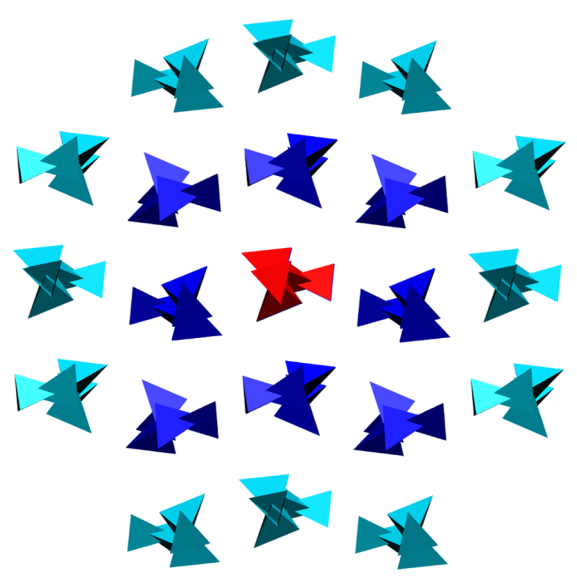

B

Figure 1. Schematic representations (with xylitol molecules in polyhedral rendering) of the crystal environment treatment in the ELMO-embedded Hirshfeld atom refinements: A) QM/ELMO and B) QM/ELMO/MM cases. Red: central QM unit; blue: ELMO subsystem; cyan: MM region.

3. Computation of structure factors. The aspherical atomic density functions $\left\{\rho_{A}(\boldsymbol{r})\right\}$ resulting from point 2 are Fourier transformed to get the corresponding thermally averaged atomic form factors, which are then summed to obtain the global calculated structure factor amplitudes $\left\{\left|F_{\boldsymbol{h}}^{\text {calc }}\right|\right\}$, with $\boldsymbol{h}$ as the generic triad of Miller indices characterizing each reflection.

4. Least-squares minimization of the statistical agreement $\chi^{2}$. The calculated and experimental structure factor amplitudes (here indicated as $\left\{\left|F_{\boldsymbol{h}}^{\text {calc }}\right|\right\}$ and $\left\{\left|F_{\boldsymbol{h}}^{\text {exp }}\right|\right\}$, respectively) are compared to obtain the statistical agreement $\chi^{2}$ :

$$
\chi^{2}=\frac{1}{N_{r}-N_{p}} \sum_{\boldsymbol{h}} \frac{\left(\eta\left|F_{\boldsymbol{h}}^{\text {calc }}\right|-\left|F_{\boldsymbol{h}}^{\text {exp }}\right|\right)^{2}}{\sigma_{\boldsymbol{h}}^{2}}
$$

where $N_{r}$ is the number of reflections taken into account, $N_{p}$ the number of parameters used in the model, and $\eta$ a scale-factor that sets the computed structure factor amplitudes on the same scale of the experimental ones. $\chi^{2}$ is least-squared minimized with respect to 
$\eta$ and the atomic structural parameters (namely, atomic coordinates and ADPs) of the chosen reference unit.

5. Check of convergence. The convergence of the refinement is checked on the atomic structural parameters: if it is achieved, the procedure is halted and a refined crystal structure is obtained, otherwise the new geometry for the reference crystal unit is used to restart the cycle and, in particular, to carry out another QM/ELMO or QM/ELMO/MM computation (see point 1 above).

The new ELMO-based embedded-HAR technique has been implemented by interfacing the quantum crystallographic software Tonto $^{55}$ (Version No. 19.12.17 v. dd352d58), which enables to perform Hirshfeld atom refinements, with an in-house modified version of the quantum chemistry package Gaussian09, ${ }^{56}$ where the QM/ELMO and QM/ELMO/MM approaches have been coded. Also Tonto (Version No. 19.12.17 v. dd352d58) was slightly modified to enable the reading of the QM/ELMO and QM/ELMO/MM formatted checkpoint files. In particular, our variants of Tonto and Gaussian09 were coupled through an in-house Bash script that follows the philosophy of the lamaGOET ${ }^{57,58}$ GUI (graphical user interface), according to which i) the geometry of the system under exam is passed from Tonto to a quantum chemical software in order to perform a QM calculation and ii) the resulting density matrix/wave function is afterwards read in Tonto for the subsequent structural refinement, repeating the procedure until convergence is reached. A similar strategy is also followed by the recently proposed NoSpherA2 system ${ }^{59}$ that allows to perform HARs through the Olex2 software $^{60,61}$ by exploiting wavefunction calculations computed by means of traditional quantum chemistry packages.

To test the refinement method described above, we considered the crystal structure of xylitol, which is characterized by strong intermolecular interactions (particularly, hydrogen bonds). The X-ray data used for the refinements were collected by Madsen and collaborators ${ }^{62}$ at 
122.4 $\mathrm{K}$ and up to a resolution of $1.22 \AA^{-1}$ (no cutoffs were applied). Four different types of Hirshfeld atom refinements were performed: i) traditional HARs without embedding, ii) HARs with crystal environment given by a cluster of point charges ( $4 \AA$ and $8 \AA$ as radii to define the clusters), iii) HARs with crystal embedding treated at QM/ELMO level (4 $\AA$ and 8 $\AA$ as radii to define the ELMO regions), iv) HARs with crystal environment described at QM/ELMO/MM level with an ELMO region up to $4 \AA$ and an MM region from 4 to $8 \AA$ (see Figure 1 and also Table S1 in the Supporting Information). Refinements (i) and (ii) were performed by exploiting the lamaGOET GUI (https://github.com/lomalaspina/lamaGOET), while (iii) and (iv) were carried out using our in-house script. In all cases, the reference crystal-unit was described at DFT-B3LYP level of theory with basis-sets cc-pVDZ and ccpVTZ. For all the QM/ELMO/MM computations, the MM subsystem was treated by interfacing our modified version of Gaussian09 with the Molecular Dynamics package AMBER $2016^{63}$ and exploiting the general AMBER force field (GAFF) ${ }^{64}$ with charges generated using the AM1-BCC model ${ }^{65,66}$. Finally, the results of the different HARs were compared to those obtained through the refinement of corresponding neutron data ${ }^{67}$ measured at $122 \mathrm{~K}$.

For the sake of completeness, figures of merits and descriptors that show the positive outcome of the performed refinements are shown in Table $\mathrm{S} 2\left(\chi^{2}, R\right.$, maximum and minimum residual density values) and Figure S3 (plots of the Meindl-Henn fractal distributions ${ }^{68}$ ). Here we will focus on the analysis of the obtained structural results.

To this regard, let us now consider the positions of the oxygen-bonded hydrogen atoms, which are also the atoms involved in strong hydrogen bond interactions with the other xylitol units in the crystal. The $\mathrm{O}-\mathrm{H}$ bond distances resulting from the different variants of HAR are shown in Figure 2. Concerning the results obtained with the cc-pVDZ basis set, it is easy to observe that the Hirshfeld atom refinement without embedding and the HARs exploiting 
clusters of point charges significantly underestimate all the neutron reference values, while all the ELMO-embedded HARs provide bond lengths that optimally agree with the neutron results. The same trends were observed when basis-set cc-pVTZ was used for the underlying quantum chemical calculations. In fact, the O-H bond distances obtained through traditional HARs without embedding or with embeddings of point charges are systematically shorter than the considered reference values, while those resulting from refinements with the crystal environment described at ELMO or ELMO/MM level are in very good agreement with the benchmark results, except for the O3-H13 bond whose length is overestimated. Furthermore, we can interestingly observe that the results do not change when the size of the embedding region is varied ( 4 or $8 \AA$ ), but only when a different strategy to treat the crystal embedding is adopted.

The results for the $\mathrm{C}-\mathrm{H}$ bond lengths are displayed in Figure $\mathrm{S} 4$ of the Supporting Information. In this case, for both the two considered basis-sets, we can see that the bond distances obtained through the different HARs are generally indistinguishable from the statistical point of view. This can be explained with the fact that most of C-H bonds in xylitol do not establish intermolecular interactions and, therefore, carrying out an embedded QM calculation is not really essential to improve the corresponding bond lengths. This is in agreement with a recent study conducted by Chodkiewicz et al., who also proposed extensions of HAR to other partitioning methods of the electron density. ${ }^{69}$ However, we can also observe that the two cases in which the distances obtained through ELMO-embedded HARs differ the most from those resulting from traditional HARs with cluster charges correspond to $\mathrm{C}-\mathrm{H}$ bonds that are involved in hydrogen bond-like contacts with the surrounding xylitol units, namely bonds C4-H4 and C1-H1B (see also Figure S5 in the Supporting Information, reporting the network of inter- and intra-molecular contacts as obtained from PLATON analysis $^{70}$ ). 


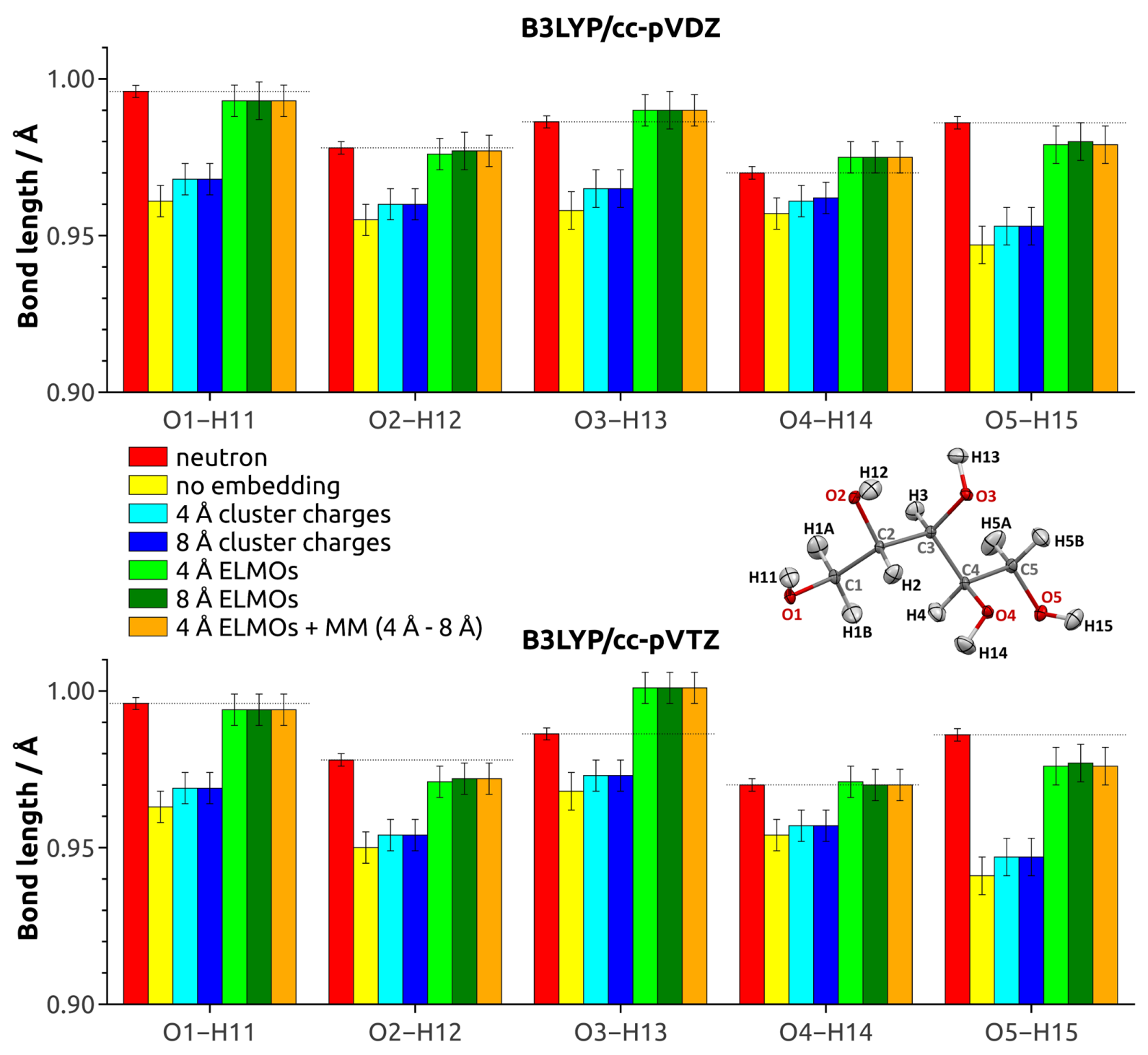

Figure 2. O-H bond lengths resulting from the performed Hirshfeld atom refinements, along with the neutron crystal structure of xylitol with specification of the atomic labels.

For a more quantitative comparison, in Table 1 we reported the mean absolute deviations (MADs) of the determined bond distances from the corresponding reference neutron values. Other than $\mathrm{C}-\mathrm{H}$ and $\mathrm{O}-\mathrm{H}$ bond lengths already discussed above, in this analysis we also specifically considered $\mathrm{H} \cdots \mathrm{A}$ (hydrogen-acceptor) distances and $\mathrm{O}-\mathrm{H} \cdots \mathrm{A}$ (oxygen-hydrogenacceptor) angles related to the hydrogen bonds established by xylitol in the crystal. Concerning the $\mathrm{O}-\mathrm{H}$ distances, regardless of the used basis-set, for HARs based on QM/ELMO and QM/ELMO/MM calculations we have MADs that are significantly lower 
(even from a statistical point of view) than those resulting from HARs without embedding or with cluster charges. For example, considering the cc-pVTZ basis-set, the mean absolute deviation associated with the Hirshfeld atom refinement without embedding amounts to 0.029(11) $\AA$, it decreases to $0.024(10) \AA$ for the HARs with clusters of point charges, and it eventually drops to $0.006(6) \AA$ when HAR is coupled with the QM/ELMO and

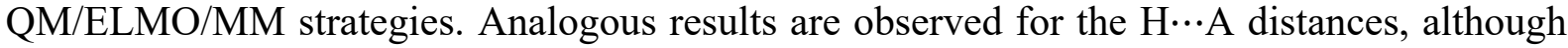
for the cc-pVTZ basis-set MADs differ by less than one standard deviation. Also for the O$\mathrm{H} \cdots \mathrm{A}$ angles, the mean absolute deviations systematically decrease as the crystal environment is described more and more accurately. The same trends are observed for the $\mathrm{C}-\mathrm{H}$ bond lengths, but, in agreement with the results already reported in Figure S4, the discrepancies associated with the ELMO-embedded refinements are not significantly lower than those corresponding to traditional HARs. Similar conclusions can be also drawn from the analysis of the mean ratios between HAR and neutron bond lengths, which are reported in the Supporting Information (see Table S3) along with the actual values of bond distances and angles associated with hydrogen bond-like contacts in the xylitol crystal structure (see Tables S4 and S5). Finally, before concluding this discussion on the statistical analyses of the obtained bond lengths, two further aspects have to be pointed out. First of all, it is again evident that it is the strategy through which the crystal environment is described during the refinement that impacts on the final results, and not the size of the embedding region (i.e., 4 and $8 \AA$ radii always give identical results). Furthermore, it is also interesting to note that the standard deviations associated with the MADs for ELMO-embedded HARs are always lower than those for the other types of refinements, thus indicating that, on average, we have a lower variability of the discrepancies from the neutron results when the crystal environment is described more accurately. 
Table 1. Mean absolute deviations of HAR distances and angles from the corresponding neutron ones, as resulting from the performed Hirshfeld atom refinements of the xylitol crystal structure. ${ }^{\text {(a) }}$

\begin{tabular}{|c|c|c|c|c|c|c|c|c|}
\hline Basis-set / Type of Embedding & \multicolumn{2}{|c|}{ C-H / A } & \multicolumn{2}{|c|}{$\mathbf{O}-\mathbf{H} / \AA$} & \multicolumn{2}{|c|}{$\mathbf{H} \cdots \mathbf{A} / \AA$} & \multicolumn{2}{|c|}{$\mathbf{O}-\mathbf{H} \cdots \mathbf{A} /^{\circ}$} \\
\hline no embedding & 0.013 & 0.010 & 0.029 & 0.014 & 0.026 & 0.014 & 0.9 & 0.7 \\
\hline $4 \AA$ cluster charges & 0.012 & 0.009 & 0.023 & 0.012 & 0.020 & 0.012 & 0.7 & 0.7 \\
\hline $8 \AA \AA$ cluster charges & 0.012 & 0.009 & 0.023 & 0.012 & 0.020 & 0.012 & 0.7 & 0.7 \\
\hline $4 \AA ̊$ ELMOs & 0.009 & 0.007 & 0.004 & 0.002 & 0.005 & 0.002 & 0.4 & 0.4 \\
\hline $8 \AA ̊$ ELMOs & 0.009 & 0.006 & 0.004 & 0.002 & 0.004 & 0.002 & 0.3 & 0.4 \\
\hline $4 \AA$ ELMOs + MM $(4 \AA-8 \AA)$ & 0.009 & 0.006 & 0.004 & 0.002 & 0.005 & 0.002 & 0.3 & 0.4 \\
\hline \multicolumn{9}{|l|}{$c c-p V T Z$} \\
\hline no embedding & 0.011 & 0.008 & 0.029 & 0.011 & 0.025 & 0.011 & 0.8 & 0.7 \\
\hline $4 \AA ̊$ cluster charges & 0.010 & 0.007 & 0.024 & 0.010 & 0.021 & 0.011 & 0.7 & 0.6 \\
\hline $8 \AA ̊$ cluster charges & 0.010 & 0.006 & 0.024 & 0.010 & 0.021 & 0.011 & 0.7 & 0.6 \\
\hline $4 \AA$ ELMOs & 0.007 & 0.005 & 0.006 & 0.006 & 0.008 & 0.004 & 0.4 & 0.2 \\
\hline $8 \AA$ ELMOs & 0.007 & 0.005 & 0.006 & 0.006 & 0.007 & 0.004 & 0.5 & 0.2 \\
\hline $4 \AA$ ELMOs + MM $(4 \AA-8 \AA)$ & 0.007 & 0.005 & 0.006 & 0.006 & 0.007 & 0.004 & 0.5 & 0.2 \\
\hline
\end{tabular}

(a) For each distance or angle, the first column reports the mean absolute discrepancies, while the second one provides the corresponding population standard deviations upon averaging.

As already mentioned above, thermal parameters of hydrogen atoms resulting from HARs do not generally seem as accurate as the obtained E-H bond lengths when they are compared to neutron reference values, although it is also important to bear in mind that differences between X-ray and neutron ADPs may be also caused by other effects, such as absorption, extinction, disorder, thermal diffuse scattering, multipole scattering and scan truncation. ${ }^{71,72}$ In this regard, we will now examine the anisotropic displacement parameters resulting from the Hirshfeld atom refinements carried out in this work with a dual goal: i) completing the analysis of the obtained structural data and, above all, ii) understanding whether the quantum mechanical treatment of the crystal environment can be also advantageous to improve the description of the (hydrogen) anisotropic displacement parameters. 
In Figures S6 and S7 we showed the refined structures of xylitol with thermal ellipsoids corresponding to the obtained ADPs. From visual inspection, except for some differences at hydrogens H13, H14 and H15, all the performed HARs provided almost identical results, with ellipsoids for the hydrogen atoms that are sometimes more elongated and differently oriented compared to the neutron reference ones. To better visualize the discrepancies between the (Xray) HAR and neutron ADPs, we also reported PEANUT plots $^{73}$ in Figures S8 and S9. The depicted differences for non-hydrogen atoms and hydrogen atoms bonded to carbons are visually identical for all the considered HAR strategies, while for hydrogen atoms bonded to oxygens the PEANUT isosurfaces corresponding to HARs with ELMO embedding are substantially different from those obtained through the other two variants of Hirshfeld atom refinement. For a more quantitative analysis, in Tables 2 and 3 we reported the mean absolute deviations of the obtained diagonal and non-diagonal ADPs from the corresponding neutron values. For non-hydrogen atoms and hydrogens bonded to carbons, all the HARs practically gave identical results. On the contrary a trend is observed for the ADPs of hydrogen atoms bonded to oxygen, thus confirming what was qualitatively observed in the PEANUT plots. In fact, especially for the diagonal elements, the MADs resulting from HARs coupled with the $\mathrm{QM} / \mathrm{ELMO}$ and QM/ELMO/MM calculations are much lower, although not statistically different, from those obtained through the other refinements. Analogous conclusions can be also drawn from the average ratios of the ADPs diagonal elements (see Table S6 in the Supporting Information). 
Table 2. Mean absolute deviations (in $\AA^{2}$ ) of the HAR diagonal elements of the ADPs from the corresponding neutron values $\left(\left\langle\left|U_{\mathrm{HAR}}^{\mathrm{ii}}-\mathrm{U}_{\mathrm{N}}^{\mathrm{ii}} \mathrm{utron}\right|\right\rangle\right)$ for different groups of atoms in xylitol. ${ }^{(\mathrm{a})}$

\begin{tabular}{|c|c|c|c|c|c|c|}
\hline Basis-set / Type of Embedding & \multicolumn{2}{|c|}{ Non-H } & \multicolumn{2}{|c|}{$\mathrm{H}$ bonded to $\mathrm{C}$} & \multicolumn{2}{|c|}{$\mathrm{H}$ bonded to $\mathrm{O}$} \\
\hline \multicolumn{7}{|l|}{$c c-p V D Z$} \\
\hline no embedding & 0.0018 & 0.0007 & 0.0031 & 0.0026 & 0.0055 & 0.0054 \\
\hline $4 \AA$ cluster charges & 0.0018 & 0.0007 & 0.0030 & 0.0027 & 0.0043 & 0.0041 \\
\hline $8 \AA$ cluster charges & 0.0018 & 0.0007 & 0.0030 & 0.0027 & 0.0042 & 0.0041 \\
\hline $4 \AA$ ELMOs & 0.0018 & 0.0007 & 0.0035 & 0.0026 & 0.0028 & 0.0022 \\
\hline $8 \AA ̊$ ELMOs & 0.0018 & 0.0007 & 0.0035 & 0.0027 & 0.0028 & 0.0022 \\
\hline $4 \AA$ ELMOs + MM (4 $\AA-8 \AA)$ & 0.0018 & 0.0007 & 0.0035 & 0.0026 & 0.0028 & 0.0022 \\
\hline \multicolumn{7}{|l|}{$c c-p V T Z$} \\
\hline no embedding & 0.0018 & 0.0007 & 0.0049 & 0.0031 & 0.0097 & 0.0066 \\
\hline $4 \AA$ cluster charges & 0.0018 & 0.0007 & 0.0048 & 0.0029 & 0.0082 & 0.0055 \\
\hline $8 \AA$ cluster charges & 0.0018 & 0.0007 & 0.0048 & 0.0029 & 0.0082 & 0.0055 \\
\hline $4 \AA ̊$ ELMOs & 0.0018 & 0.0007 & 0.0050 & 0.0028 & 0.0049 & 0.0036 \\
\hline $8 \AA ̊$ ELMOs & 0.0018 & 0.0007 & 0.0050 & 0.0028 & 0.0048 & 0.0037 \\
\hline $4 \AA$ ELMOs + MM $(4 \AA-8 \AA)$ & 0.0018 & 0.0007 & 0.0050 & 0.0028 & 0.0048 & 0.0037 \\
\hline
\end{tabular}

(a) For each group of atoms, the first column reports the mean absolute discrepancies, while the second one provides the corresponding population standard deviations upon averaging. 
Table 3. Mean absolute deviations (in $\AA^{2}$ ) of the HAR non-diagonal elements of the ADPs from the corresponding neutron values $\left(\left\langle\left|\mathrm{U}_{\mathrm{HAR}}^{\mathrm{ij}}-\mathrm{U}_{\mathrm{Neutron}}^{\mathrm{ij}}\right|\right\rangle\right.$, with $\left.i \neq j\right)$ for different groups of atoms in xylitol. ${ }^{(a)}$

\begin{tabular}{|c|c|c|c|c|c|c|}
\hline Basis-set / Type of Embedding & \multicolumn{2}{|c|}{ Non-H } & \multicolumn{2}{|c|}{$\mathrm{H}$ bonded to $\mathrm{C}$} & \multicolumn{2}{|c|}{$\mathrm{H}$ bonded to $\mathrm{O}$} \\
\hline \multicolumn{7}{|l|}{$c c-p V D Z$} \\
\hline no embedding & 0.0002 & 0.0002 & 0.0035 & 0.0027 & 0.0042 & 0.0027 \\
\hline $4 \AA$ cluster charges & 0.0002 & 0.0002 & 0.0035 & 0.0027 & 0.0039 & 0.0025 \\
\hline $8 \AA ̊$ cluster charges & 0.0002 & 0.0002 & 0.0035 & 0.0027 & 0.0039 & 0.0025 \\
\hline $4 \AA ̊$ ELMOs & 0.0002 & 0.0002 & 0.0035 & 0.0028 & 0.0036 & 0.0021 \\
\hline $8 \AA ̊$ ELMOs & 0.0002 & 0.0002 & 0.0035 & 0.0028 & 0.0036 & 0.0021 \\
\hline $4 \AA$ ELMOs + MM $(4 \AA$ & 0.0002 & 0.0002 & 0.0035 & 0.0028 & 0.0036 & 0.0021 \\
\hline \multicolumn{7}{|l|}{$c c-p V T Z$} \\
\hline no embedding & 0.0002 & 0.0002 & 0.0031 & 0.0019 & 0.0042 & 0.0038 \\
\hline $4 \AA$ cluster charges & 0.0002 & 0.0002 & 0.0030 & 0.0019 & 0.0037 & 0.0035 \\
\hline $8 \AA$ A cluster charges & 0.0002 & 0.0002 & 0.0030 & 0.0019 & 0.0037 & 0.0035 \\
\hline $4 \AA ̊$ ELMOs & 0.0002 & 0.0002 & 0.0032 & 0.0018 & 0.0032 & 0.0030 \\
\hline $8 \AA ̊$ ELMOs & 0.0002 & 0.0002 & 0.0032 & 0.0018 & 0.0032 & 0.0030 \\
\hline $4 \AA$ ELMOs + MM (4 A - $8 \AA)$ & 0.0002 & 0.0002 & 0.0032 & 0.0018 & 0.0032 & 0.0030 \\
\hline
\end{tabular}

(a) For each group of atoms, the first column reports the mean absolute discrepancies, while the second one provides the corresponding population standard deviations upon averaging.

To rationalize all the above-discussed results, we finally decided to compare the electron densities associated with the quantum mechanical calculations underlying the performed Hirshfeld atoms refinements. They were computed on the IAM structure of xylitol, which was the starting geometry for all the performed HARs. In this way, the obtained electron distributions were directly comparable. Furthermore, it is worth noting that the largest structural changes in HARs are usually observed at the first iteration, which is therefore the most crucial one and usually has the largest influence on the final outcome of the refinement. The comparison of the electron densities computed on the starting IAM structure should thus allow us to explain the main reasons behind the different results obtained with the HAR variants considered in this study. To this aim, using as reference the electron distribution 
computed at B3LYP level without embedding, in Figure 3 we displayed all the electron density differences obtained with basis-set cc-pVTZ (see Figure S10 in the Supporting Information for the analogous plots obtained with basis-set cc-pVDZ). We can observe that, for all the refinements with embedding, the differences are mainly localized on all the hydroxy groups of xylitol. They are significantly more evident in the ELMO-embedded cases, for which we also notice some non-negligible variations in correspondence of the C4-H4 and C1-H1B bonds whose lengths were indeed those that changed the most among the C-H ones when ELMO-embedded HARs were performed. Concerning the density differences detected at the hydroxy groups for the ELMO-embedded calculations, we have charge depletions at the hydrogens and charge accumulations at the oxygens (particularly, in the directions pointing towards the surroundings hydrogens atoms), thus indicating charge density shifts ascribable to the hydrogen bond interactions. Therefore, it is clear that the ELMO embeddings allowed to significantly and successfully account for those electron density variations that are due to intermolecular interactions with the crystal environment, thus leading to $\mathrm{O}-\mathrm{H}$ (and to some CH) refined bond lengths that are in much better agreement with the neutron reference values.

To summarize, in this Letter we have proposed an improved version of the Hirshfeld atom refinement technique consisting in the coupling of the original HAR algorithm with the recently proposed embedding methods QM/ELMO and QM/ELMO/MM. Our preliminary validation tests on xylitol have shown that, through its more rigorous description of the crystal environment, the new version of Hirshfeld atom refinement is fundamental to obtain significantly more accurate positions of hydrogen atoms, especially if they are directly involved in intermolecular interaction networks. From the test calculations it also emerged that a $4 \AA$ ELMO embedding is generally enough to obtain completely satisfactory results, without the need of also including an outer Molecular Mechanics layer. Further tests will be performed to completely assess all capabilities and limitations of the new approach. However, 
borrowing the concept of Jacob's ladder used for the classification of exchange-correlation functionals in DFT,${ }^{74}$ the strategy proposed in this work could also be seen as an attempt of introducing a new rung in the stairway that connects IAM to real crystal structures, thus paving the way to future couplings of HAR with other fully QM embedding methods of quantum chemistry ${ }^{75,76}$ in order to carry out more and more accurate X-ray based structural refinements.

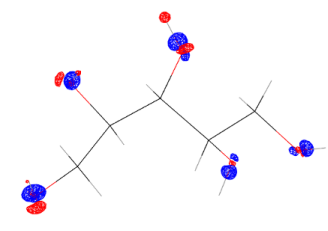

$4 \AA$ cluster charges

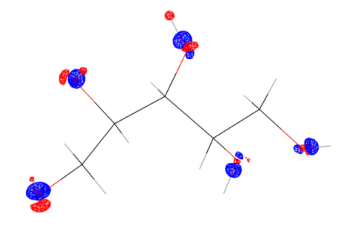

$8 \AA$ cluster charges
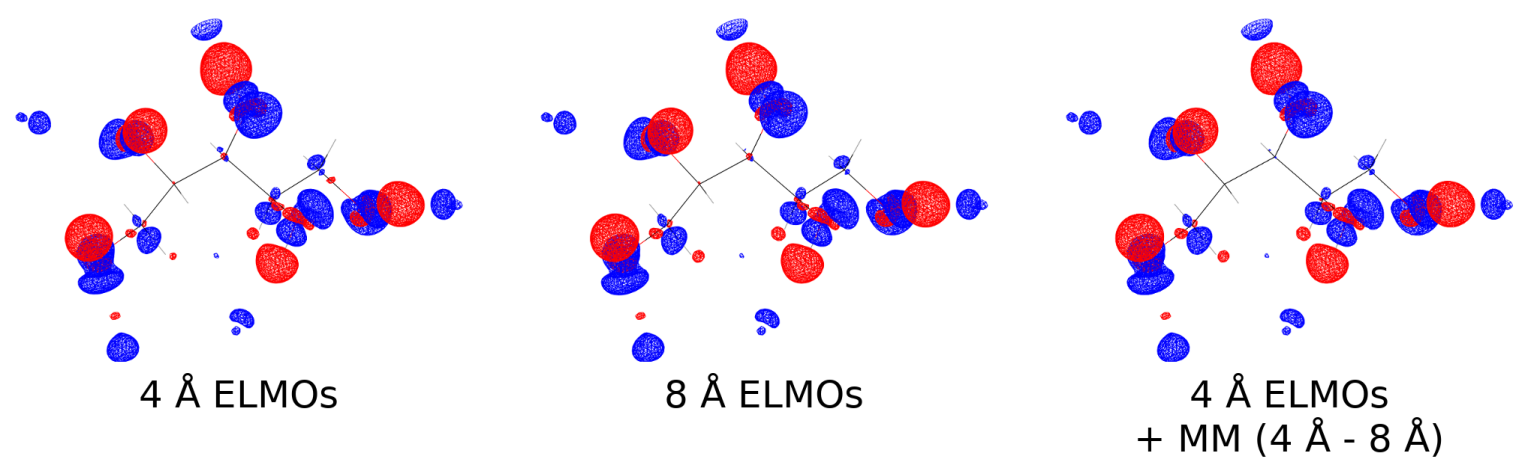

Figure 3. Electron density differences for xylitol obtained for the different embedding schemes compared to the calculation without embedding. All the electron densities were computed on the IAM structure exploiting the cc-pVTZ basis-set. Contour level: $0.003 \mathrm{e} \mathrm{bohr}^{-3}$; Colors: blue (positive) and red (negative). Orientation of the xylitol molecules as in Figure 2. 


\section{AUTHORS INFORMATION}

\section{Corresponding Author}

Alessandro Genoni (A.G.), Université de Lorraine \& CNRS, Laboratoire de Physique et Chimie Théoriques (LPCT), 1 Boulevard Arago, F-57078 Metz, France; Phone: +33 (0)3 72 7491 70; Fax: +33 (0)3 727491 87. E-mail: Alessandro.Genoni@ univ-lorraine.fr.

\section{Notes}

The authors declare no competing financial interests.

\section{ACKNOWLEDGMENTS}

The French Research Agency (ANR) is gratefully acknowledged for financial support of this work through the Young Investigator Project QuMacroRef (Grant No. ANR-17-CE29-000501). The High-Performance Computing Center EXPLOR of the University of Lorraine is thanked for providing computing time through the projects 2019CPMXX0966, 2019CPMXX0886 and 2019CPMXX1332. Fabien Pascale is also acknowledged for the setup and maintenance of our local cluster, which was used to perform most of the calculations reported in this paper. Hans-Beat Bürgi is thanked for providing us the PENAUT software and for useful discussions.

\section{ASSOCIATED CONTENT}

Supporting Information. Details about theory, transfer and libraries of extremely localized molecular orbitals (with Figure S1 reporting some examples of plotted ELMOs and Figure S2 showing the schematic representation of reference frames and atomic triads required for the ELMOs rotation). Details about the QM/ELMO and QM/ELMO/MM methods. Figure S3 displaying the Meindl-Henn plots associated with the different Hirshfeld atom refinements. Figure S4 showing the $\mathrm{C}-\mathrm{H}$ bond lengths of xylitol resulting from the performed HARs. 
Figure S5 depicting the network of inter- and intra-molecular contacts in the xylitol crystal structure. Figures S6 and S7 reporting the refined structures of xylitol with thermal ellipsoids. Figures S8 and S9 depicting the PEANUT plots of the differences between (X-ray) HAR and neutron ADPs. Figure S10 showing electron density differences computed on the IAM structure with basis-set cc-pVDZ. Table S1 reporting the number of atoms and basis functions involved in the ELMO-embedded Hirshfeld atom refinements. Table S2 reporting the values of the figures of merit associated with the different HARs. Table S3 providing the mean ratios between HAR and neutron distances/angles. Tables S4 and S5 reporting the actual values of bond distances and angles associated with inter- and intra-molecular contacts in the xylitol crystal structures. Table S6 giving the average ratios between HAR and neutron values of the ADPs diagonal elements. (PDF)

Crystallographic information files (cif files) and files containing experimental and calculated structure factors (fco files) associated with all the performed Hirshfeld atom refinements of the xylitol crystal structure. (ZIP)

This material is available free of charge via the Internet at http://pubs.acs.org/.

\section{DATA AVAILABILITY}

The xylitol structures obtained in the present study through the ELMO-embedded HAR technique with a $4 \AA$ ELMO-layer (cc-pVDZ and cc-pVTZ basis-sets for the underlying quantum chemical calculations) have been deposited at the Cambridge Crystallographic Data Centre (CCDC) under the deposition numbers 2044846 and 2044847. This data can be obtained free of charge via www.ccdc.cam.ac.uk/data_request/cif. All models for all refinements plus the measured structure factors have been deposited with this journal in addition to the CCDC deposition to ensure reproducibility of the study. 


\section{REFERENCES}

${ }^{1}$ Halliwell, B.; Gutteridge, J. Free Radicals in Biology and Medicine, $3{ }^{\text {rd }}$ Ed; Oxford University Press: Oxford, UK, 1999.

${ }^{2}$ Hynes, J. T.; Klinman, J.; Limbach, H.-H.; Schowen, R. Hydrogen Transfer Reactions, Vols. 1-4. Wiley-VCH: Weinheim, 2007.

${ }^{3}$ Jena, P. Materials for hydrogen storage: Past, present, and future. J. Phys. Chem. Lett. 2011, 2, 206211.

${ }^{4}$ Desiraju, G. Crystal Engineering - The Design of Organic Solids. Elsevier: Amsterdam, 1989.

${ }^{5}$ Desiraju, G. R.; Steiner, T. The Weak Hydrogen Bond in Structural Chemistry and Biology. Oxford University Press: Oxford, UK, 1999.

${ }^{6}$ Hoser, A. A.; Madsen, A. Ø. Dynamic quantum crystallography: lattice dynamical models refined against diffraction data. II. Applications to L-alanine, naphthalene and xylitol. Acta Cryst. A 2017, 73, $102-114$.

${ }^{7}$ A. H. Compton, The Distribution of Electrons in Atoms. Nature 1915, 95, 343-344.

${ }^{8}$ Allen, F. H.; Watson, D. G.; Brammer, L.; Orpen, A. G.; Taylor, R. International Tables for Crystallography, Vol. C, 1st online Ed. International Union of Crystallography: Chester, UK, 2006; Chapter 9.5, pp 790-811.

${ }^{9}$ Allen, F. H.; Bruno, I. J. Bond lengths in organic and metal-organic compounds revisited: X-H bond lengths from neutron diffraction data. Acta Cryst. B 2010, 66, 380-386.

${ }^{10}$ Grabowsky, S.; Genoni, A.; Bürgi, H.-B. Quantum Crystallography. Chem. Sci. 2017, 8, 4159-4176.

${ }^{11}$ Genoni, A.; Bučinský, L.; Claiser, N.; Contreras-García, J.; Dittrich, B.; Dominiak, P. M.; Espinosa, E.; Gatti, C.; Giannozzi, P.; Gillet et al. Quantum Crystallography: Current Developments and Future Perspectives. Chem. Eur. J. 2018, 24, 10881-10905.

${ }^{12}$ Massa, L.; Matta, C. F. Quantum Crystallography: A perspective. J. Comput. Chem. 2018, 39, 10211028. 
${ }^{13}$ Genoni, A.; Macchi, P. Quantum Crystallography in the Last Decade: Developments and Outlooks. Crystals 2020, 10, 473.

${ }^{14}$ Grabowsky, S.; Genoni, A.; Thomas, S. P.; Jayatilaka, D. The Advent of Quantum Crystallography: Form and Structure Factors from Quantum Mechanics for Advanced Structure Refinement and Wavefunction Fitting. In Structure and Bonding. $21^{\text {st }}$ Century Challenges in Chemical Crystallography 2 - Structural Correlations and Data Interpretation; Mingos, D. M. P., Rathby, P., Eds.; Springer: Berlin, Heidelberg; DOI: 10.1007/430_2020_62.

${ }^{15}$ Stewart, R. F.; Davidson, E. R.; Simpson, W. T. Coherent X-Ray Scattering for the Hydrogen Atom in the Hydrogen Molecule. J. Chem. Phys. 1965, 42, 3175-3187.

${ }^{16}$ Stewart, R. F. Electron Population Analysis with Rigid Pseudoatoms. Acta Cryst. A 1976, 32, $565-574$.

${ }^{17}$ Hansen, N. K.; Coppens, P. Testing Aspherical Atom Refinements on Small-Molecule Data Sets. Acta Cryst. A 1978, 34, 909-921.

${ }^{18}$ Dittrich, B.; Hübschle, C. B.; Messerschmidt, M.; Kalinowski, R.; Girnt, D.; Luger, P. The invariom model and its application: refinement of D,L-serine at different temperatures and resolution. Acta Cryst. A 2005, 61, 314-320.

${ }^{19}$ Dittrich, B.; Weber, M.; Kalinowski, R.; Grabowsky, S.; Hübschle, C. B.; Luger, P. How to easily replace the independent atom model - the example of bergenin, a potential anti-HIV agent of traditional Asian medicine. Acta Cryst. B 2009, 65, 749-756.

${ }^{20}$ Bendeif, E.; Jelsch, C. The experimental library multipolar atom model refinement of L-aspartic acid. Acta Cryst. C 2007, 63, o361-0364

${ }^{21}$ Bąk, J. M.; Domagała, S.; Hübschle, C.; Jelsch, C.; Dittrich, B.; Dominiak, P. M. Verification of Structural and Electrostatic Properties Obtained by the Use of Different Pseudoatom Databases. Acta Cryst. A 2011, 67, 141-153.

${ }^{22}$ Dadda, N.; Nassour, A.; Guillot, B.; Benali-Cherif, N.; Jelsch, C.; Charge-density analysis and electrostatic properties of 2-carboxy-4-methylanilinium chloride monohydrate obtained using a multipolar and a spherical-charges model. Acta Cryst. A 2012, 68, 452-463. 
${ }^{23}$ Jha, K. K.; Gruza, B.; Kumar, P.; Chodkiewicz, M. L.; Dominiak, P. M. TAAM: A reliable and user friendly tool for hydrogen atom location using routine X-ray diffraction data. Acta Cryst. B 2020, 76, 296-306.

${ }^{24}$ Destro, R.; Merati, F. Bond lengths, and beyond. Acta Cryst. B 1995, 51, 559-570.

${ }^{25}$ Jayatilaka, D.; Dittrich, B. X-ray structure refinement using aspherical atomic density functions obtained from quantum-mechanical calculations. Acta Cryst. A 2008, 64 383-393.

${ }^{26}$ Capelli, S.; Bürgi, H.-B.; Dittrich, B.; Grabowsky, S.; Jayatilaka, D. Hirshfeld atom refinement. IUCrJ 2014, 1, 361-379.

${ }^{27}$ Woińska, M.; Jayatilaka, D.; Spackman, M. A.; Edwards, A. J.; Dominiak, P. M.; Woźniak, K.; Nishibori, E.; Sugimoto, K.; Grabowsky, S. Hirshfeld atom refinement for modeling strong hydrogen bonds. Acta Cryst. A 2014, 70, 483-498.

${ }^{28}$ Woińska, M.; Grabowsky, S.; Dominiak, P. M.; Woźniak, K.; Jayatilaka, D. Hydrogen atoms can be located accurately and precisely by X-ray crystallography. Sci. Adv. 2016, 2, e1600192.

${ }^{29}$ Wall, M. E. Quantum crystallographic charge density of urea. IUCrJ 2016, 3, 237-246.

${ }^{30}$ Fugel, M.; Jayatilaka, D.; Hupf, E.; Overgaard, J.; Hathwar, V. R.; Macchi, P.; Turner, M. J.; Howard, J. A. K.; Dolomanov, O. V.; Puschmann, H. et al. Probing the accuracy and precision of Hirshfeld atom refinement with HARt interfaced with Olex2. IUCrJ 2018, 5, 32-44.

${ }^{31}$ Hirshfeld, F. L. XVII. Spatial Partitioning of Charge Density. Isr. J. Chem. 1977, 16, 198-201.

${ }^{32}$ Hirshfeld, F. L. Bonded-Atom Fragments for Describing Molecular Charge Densities. Theoret. Chim. Acta 1977, 44, 129-138.

${ }^{33}$ Köhler, C.; Lübben, J.; Krause, L.; Hoffmann, C.; Herbst-Irmer, R.; Stalke, D. Comparison of different strategies for modelling hydrogen atoms in charge density analyses. Acta Cyst. B 2019, 75, 434-441.

${ }^{34}$ Malaspina, L. A.; Hoser, A. A.; Edwards, A. J.; Woińska, M.; Turner, M. J.; Price, J. R.; Sugimoto, K.; Nishibori, E.; Bürgi, H.-B.; Jayatilaka, D. et al. Hydrogen atoms in bridging positions from 
quantum crystallographic refinements: influence of hydrogen atom displacement parameters on geometry and electron density. CrystEngComm 2020, 22, 4778-4789.

${ }^{35}$ Madsen, A. Ø. SHADE web server for estimation of hydrogen anisotropic displacement parameters. J. Appl. Cryst. 2006, 39, 757-758.

${ }^{36}$ Madsen, A. Ø. Hoser, A. A. SHADE3 server: a streamlined approach to estimate H-atom anisotropic displacement parameters using periodic ab initio calculations or experimental information. J. Appl. Cryst. 2014, 47, 2100-2104.

${ }^{37}$ Wieduwilt, E. K.; Macetti, G.; Malaspina, L. A.; Jayatilaka, D.; Grabowsky, S.; Genoni, A. PostHartree-Fock methods for Hirshfeld atom refinement: are they necessary? Investigation of a strongly hydrogen-bonded molecular crystal. J. Mol. Struct. 2020, 127934.

${ }^{38}$ Meyer, B.; Guillot, B.; Ruiz-Lopez, M. F.; Genoni, A. Libraries of Extremely Localized Molecular Orbitals. 1. Model Molecules Approximation and Molecular Orbitals Transferability. J. Chem. Theory. Comput. 2016, 12, 1052-1067.

${ }^{39}$ Meyer, B.; Guillot, B.; Ruiz-Lopez, M. F.; Jelsch, C.; Genoni, A. Libraries of Extremely Localized Molecular Orbitals. 2. Comparison with the Pseudoatoms Transferability. J. Chem. Theory. Comput. 2016, $12,1068-1081$

${ }^{40}$ Meyer, B.; Genoni, A. Libraries of Extremely Localized Molecular Orbitals. 3. Construction and Preliminary Assessment of the New Databanks. J. Phys. Chem. A 2018, 122, 8965-8981.

${ }^{41}$ Stoll, H.; Wagenblast, G.; Preuss, H. On the Use of Local Basis Sets for Localized Molecular Orbitals. Theoret. Chim. Acta 1980, 57, 169-178.

${ }^{42}$ Fornili, A.; Sironi, M.; Raimondi, M. Determination of extremely localized molecular orbitals and their application to quantum mechanics/molecular mechanics methods and to the study of intramolecular hydrogen bonding. J. Mol. Struct. (THEOCHEM) 2003, 632, 157-172.

${ }^{43}$ Genoni, A.; Sironi, M. A Novel Approach to Relax Extremely Localized Molecular Orbitals: the Extremely Localized Molecular Orbital-Valence Bond Method. Theor. Chem. Acc. 2004, 112, 254262. 
${ }^{44}$ Genoni, A.; Fornili, A.; Sironi, M. Optimal Virtual Orbitals to Relax Wave Functions Built Up with Transferred Extremely Localized Molecular Orbitals. J. Comput. Chem. 2005, 26, 827-835.

${ }^{45}$ Genoni, A.; Ghitti, M.; Pieraccini, S.; Sironi, M. A novel extremely localized molecular orbitals based technique for the one-electron density matrix computation. Chem. Phys. Lett. 2005, 415, 256260.

${ }^{46}$ Sironi, M.; Genoni, A.; Civera, M.; Pieraccini, S.; Ghitti, M. Extremely Localized Molecular Orbitals: Theory and Applications. Theor. Chem. Acc. 2007, 117, 685-698.

${ }^{47}$ Sironi, M.; Ghitti, M.; Genoni, A.; Saladino, G.; Pieraccini, S. DENPOL: A new program to determine electron densities of polypeptides using extremely localized molecular orbitals. $J$ Mol. Struct. (THEOCHEM) 2009, 898, 8-16.

${ }^{48}$ Malaspina, L. A.; Wieduwilt, E. K.; Bergmann, J.; Kleemiss, F.; Meyer, B.; Ruiz-López, M. F.; Pal, R.; Hupf, E.; Beckmann, J.; Piltz, R. O. et al. Fast and Accurate Quantum Crystallography: from Small to Large, from Light to Heavy, J. Phys. Chem. Lett. 2019, 10, 6973-6982.

${ }^{49}$ Bergmann, J.; Davidson M.; Oksanen, E.; Ryde, U.; Jayatilaka, D. fragHAR: towards ab initio quantum-crystallographic X-ray structure refinement for polypeptides and proteins. IUCrJ 2020, 7, 158-165.

${ }^{50}$ Zhang, D. W.; Zhang, J. Z. H. Molecular fractionation with conjugate caps for full quantum mechanical calculation of protein-molecule interaction energy. J. Chem. Phys. 2003, 119, 3599-3605.

${ }^{51}$ Macetti, G.; Genoni, A. Quantum Mechanics/Extremely Localized Molecular Orbital Method: A Fully Quantum Mechanical Embedding Approach for Macromolecules. J. Phys. Chem. A 2019, 123, 9420-9428.

${ }^{52}$ Macetti, G.; Wieduwilt, E. K.; Assfeld, X.; Genoni. A. Localized Molecular Orbital-Based Embedding Scheme for Correlated Methods. J. Chem. Theory Comput. 2020, 16, 3578-3596.

${ }^{53}$ Macetti, G.; Genoni, A. Quantum Mechanics/Extremely Localized Molecular Orbital Embedding Strategy for Excited States: Coupling to Time-Dependent Density Functional Theory and Equation-ofMotion Coupled Cluster. J. Chem. Theory Comput. 2020, 16, 7490-7506. 
${ }^{54}$ Macetti, G.; Genoni, A. Three-Layer Multiscale Approach Based on Extremely Localized Molecular Orbitals to Investigate Enzyme reactions, in preparation.

${ }^{55}$ Jayatilaka, D; Grimwood, D. J. Tonto: A Fortran Based Object-Oriented System for Quantum Chemistry and Crystallography. In Computational Science - ICCS 2003; Sloot, P. M. A., Abramson, D., Bogdanov, A. V., Dongarra, J. J., Zomaya, A. Y., Gorbachev, Y. E., Eds.; Springer-Verlag: Berlin \& Heidelberg, 2003; Chapter 4, pp 142-151.

${ }^{56}$ Frisch, M. J.; Trucks, G. W.; Schlegel, H. B.; Scuseria, G. E.; Robb, M. A.; Cheeseman, J. R.; Scalmani, G.; Barone, V.; Mennucci, B.; Petersson, G. A. et al. Gaussian 09, Revision D.01; Gaussian, Inc., Wallingford, CT, USA, 2009.

${ }^{57}$ Malaspina, L. A.; Genoni, A.; Grabowsky, S. lamaGOET: an Interface for Quantum Crystallography. J. Appl. Cryst., under revision.

${ }^{58}$ Malaspina, L. A.; Genoni, A.; Jayatilaka, D.; Turner, M. J.; Sugimoto, K.; Nishibori, E.; Grabowsky, S. The advanced treatment of hydrogen bonding in quantum crystallography. J. Appl. Cryst., under revision.

${ }^{59}$ Kleemiss, F.; Dolomanov, O. V.; Bodensteiner, M.; Peyerimhoff, N.; Midgley, L.; Borhis, L. J.; Genoni, A.; Malaspina, L. A.; Jayatilaka, D.; Spencer, J. L. et al. Accurate Crystal Structures and Chemical Properties from NoSpherA2. Chem. Sci., 2021, DOI:10.139/D0SC05526C.

${ }^{60}$ Dolomanov, O. V.; Bourhis, L. J.; Gildea, R. J.; Howard, J. A. K.; Puschmann, H. Olex2: a complete structure solution, refinement and analysis program. J. Appl. Cryst. 2009, 42, 339-341.

${ }^{61}$ Bourhis, L. J.; Dolomanov, O. V.; Gildea, R. J.; Howard, J. A. K.; Puschmann, H. The anatomy of a comprehensive constrained, restrained refinement program for the modern computing environment Olex2 dissected. Acta Cryst. A 2015, 71, 59-75.

${ }^{62}$ Madsen, A. Ø.; Sørensen, H. O.; Flensburg, C.; Stewart, R. F.; Larsen, S. Modeling of the nuclear parameters for $\mathrm{H}$ atoms in X-ray charge-density studies. Acta Cryst. A 2004, 60, 550-561. 
${ }^{63}$ Case, D. A.; Betz, R. M.; Cerutti, D. S.; Cheatham, T. E., III; Darden, T. A.; Duke, R. E.; Giese, T. J.; Gohlke, H.; Goetz, A. W.; Homeyer, N. et al. AMBER 2016; University of California San Francisco: San Francisco, CA, USA, 2016.

${ }^{64}$ Wang, J.; Wolf, R. M.; Caldwell, J. W.; Kollman, P. A.; Case, D. A. Development and Testing of a General Amber Force Field. J. Comput. Chem. 2004, 25, 1157-1173.

${ }^{65}$ Jakalian, A.; Bush, B. L.; Jack, D. B.; Bayly, C. I. Fast, efficient generation of high-quality atomic charges. AM1-BCC model: I. Method. J. Comput. Chem. 2000, 21, 132-146.

${ }^{66}$ Jakalian, A.; Jack, D. B.; Bayly, C.I. Fast, efficient generation of high-quality atomic charges. AM1BCC model: II. Parameterization and validation. J. Comput. Chem. 2002, 23, 1623-1641.

${ }^{67}$ Madsen, A. Ø.; Mason, S.; Larsen, S. A neutron diffraction study of xylitol: derivation of mean square internal vibrations for $\mathrm{H}$ atoms from a rigid-body description. Acta Cryst. B 2003, 59, 653-663.

${ }^{68}$ Meindl, K.; Henn, J. Foundations of residual-density analysis, Acta Cryst. A 2008, 64, 404-418.

${ }^{69}$ Chodkiewicz, M. L.; Woińska, M.; Wozńiak, K. Hirshfeld atom like refinement with alternative electron density partitions. IUCrJ 2020, 7, 1199-1215.

${ }^{70}$ Spek, A. L. Single-crystal structure validation with the program PLATON. J. Appl. Cryst. 2003, 36, 7-13.

${ }^{71}$ Blessing, R. H. On the Differences Between X-ray and Neutron Thermal Vibration Parameters. Acta Cryst. B 1995, 51, 816-823.

${ }^{72}$ Larsen, F. K. Diffraction studies of crystals at low temperatures - crystallography below 77 K. Acta Cryst. B 1995, 51, 468-482.

${ }^{73}$ Hummel, W.; Hauser, J.; Bürgi, H.-B. PEANUT: Computer graphics program to represent atomic displacement parameters. J. Mol. Graph. 1990, 8, 214-220.

${ }^{74}$ Perdew, J. P.; Schmidt, K. Jacob's ladder of density functional approximations for the exchangecorrelation energy. AIP Conference Proceedings 2001, 577, 1-20.

${ }^{75}$ Wesolowski, T. A.; Shedge, S.; Zhou, X. Frozen-Density Embedding Strategy for Multilevel Simulations of Electronic Structure. Chem. Rev. 2015, 115, 5891-5928. 
${ }^{76}$ Lee, S. J. R.; Welborn, M.; Manby, F. R.; Miller, T. F., III Projection-Based Wavefunction-in-DFT Embedding. Acc. Chem. Res. 2019, 52, 1359-1368. 